\title{
Enhanced and Polarization Dependent Coupling for Photoaligned Liquid Crystalline Conjugated Polymer Microcavities
}

\author{
Florian Le Roux, ${ }^{*, \dagger}$ Robert A. Taylor, ${ }^{\dagger}$ and Donal D. C. Bradley ${ }^{*, \dagger}, \dagger$ \\ $\dagger$ Department of Physics, University of Oxford, Parks Road, Oxford, OX1 3PU, U.K. \\ $\ddagger$ Physical Science and Engineering Division, King Abdullah University of Science and \\ Technology, Thuwal, 23955-6900, Saudi Arabia
}

E-mail: florian.leroux@physics.ox.ac.uk; donal.bradley@kaust.edu.sa

\begin{abstract}
Here we report the fabrication and optical characterization of organic microcavities containing liquid-crystalline conjugated polymers (LCCPs): poly(9,9-dioctylfluoreneco-benzothiadiazole) (F8BT), poly(9,9-dioctylfluorene) (PFO) and poly (2,7-(9,9-dihexyl fluorene)-co-bithiophene) (F6T2) aligned on top of a thin transparent Sulfuric Dye 1 (SD1) photoalignment layer. We extract the optical constants of the aligned films using variable angle spectroscopic ellipsometry and fabricate metallic microcavities in which the ultrastrong coupling regime is manifest both for the aligned and non-aligned LCCPs. Transition dipole moment alignment enables a systematic increase in the interaction strength, with unprecedented solid-state Rabi splittings of up to $1.80 \mathrm{eV}$, the first to reach energies comparable to those in the visible spectrum. With an optical gap of $2.79 \mathrm{eV}$ for F6T2 this gives the highest-to-date organic microcavity coupling ratio, $65 \%$. We also demonstrate that the coupling strength is polarization-dependent with bright polariton photoluminescence for TE polarization parallel to the polymer
\end{abstract}


chains and either no emission or weakly coupled emission from the corresponding TM polarization.

\section{Keywords}

ultrastrong coupling, exciton-polaritons, conjugated polymer microcavities, organic semiconductors, liquid crystalline conjugated polymers 
The strong coupling (SC) regime in the solid-state is entered ${ }^{1}$ when the interaction between the electric component of a confined electromagnetic field and the excitations present within a semiconductor becomes sufficiently intense that their original energy levels are replaced by so-called polariton hybrid states of light and matter, separated by a Rabi splitting energy $\hbar \Omega_{R}$. Organic semiconductor Frenkel excitons are an interesting alternative to the more traditional Wannier excitons seen in III-V inorganic semiconductors for the study of exciton-polaritons thanks to their large binding energies $\left(E_{B} \sim 0.5 \pm 0.25 \mathrm{eV}^{2,3}\right)$ which allow room-temperature observation of varied phenomena including Bose-Einstein condensation, ${ }^{4,5}$ superfluidity of light ${ }^{6}$ and optical logic. ${ }^{7}$ Their large intrinsic oscillator strengths ${ }^{8}$ combined with the small mode volumes $V_{\mathrm{m}}$ of metallic microcavities ${ }^{9}$ have enabled $\hbar \Omega_{\mathrm{R}} \geq 1$ $\mathrm{eV},{ }^{10-13}$ with values up to $\hbar \Omega_{\mathrm{R}}=1.12 \mathrm{eV} \cdot{ }^{14,15}$ This splitting is directly comparable to the exciton transition energy $\hbar \omega_{\text {ex }}$ and yields normalized coupling ratios $g=\frac{\Omega_{\mathrm{R}}}{\omega_{\mathrm{ex}}} \geq 20 \%$, thereby crossing into ultrastrong coupling (USC), an interaction space that has received great recent attention, with attractive research perspectives and multiple emerging applications. ${ }^{16,17}$ Experimental realizations of increasingly higher coupling ratios have also been reported for inorganic semiconductor based intersubband polaritons, ${ }^{18}$ and other physical systems, including superconducting circuits, ${ }^{19}$ Landau polaritons ${ }^{20}$ and plasmonic picocavities interacting with vibrational degrees of freedom of individual molecules. ${ }^{21}$

For an ensemble of Frenkel excitons inside a cavity for which both inhomogeneous (nonradiative broadening) and homogeneous broadening (radiative broadening and cavity damping) are small compared with the observed $\hbar \Omega_{\mathrm{R}}:^{22-24}$

$$
\hbar \Omega_{\mathrm{R}} \approx 2 \boldsymbol{\mu} \cdot \boldsymbol{E} \sqrt{\frac{N \hbar \omega_{\mathrm{ex}}}{2 \epsilon_{\mathrm{eff}} V_{\mathrm{m}}}}
$$

where $\boldsymbol{\mu}$ is the transition dipole moment, $\boldsymbol{E}$ the electric field, $N$ the number of molecules, $\hbar \omega_{\mathrm{ex}}$ the exciton transition energy, $\epsilon_{\mathrm{eff}}$ the cavity effective permittivity and $V_{\mathrm{m}}$ the cavity mode volume.

One way to increase the value of the coupling ratio is to work with lower energy exci- 
tons, as done by Barachati et al. ${ }^{25}$ resulting in a then record $g=62 \%$. This approach is inherently accompanied by a reduction of $\hbar \Omega_{\mathrm{R}}$ compared with the use of excitons lying at higher energies with equivalent oscillator strengths. The alternative is to look to increase $\hbar \Omega_{\mathrm{R}}$; The most direct routes to achieve this include (i) increasing $N$, which although generally not straightforward can be done, for example, by reducing the bulkiness of conjugated polymer solubilizing groups ${ }^{26}$ (ii) increasing $\boldsymbol{\mu}$ through conformational control ${ }^{27,28}$ or a photo-switchable configuration change, ${ }^{9}$ and/or (iii) increasing $\boldsymbol{\mu} . \boldsymbol{E}$, a phenomenom observed along the preferential axes of organic crystals. ${ }^{29}$ In the latter case, uniaxial orientation has been shown to enhance conjugated polymer thin film refractive index (and correspondingly transition dipole moment) in the direction parallel to the chain orientation axis ${ }^{30,31}$ yielding an enhanced dot product for a suitable polarization of $\boldsymbol{E}$. This offers a clear route to enhancing $\hbar \Omega_{\mathrm{R}}$ that is demonstrated below, using a photoalignment process to achieve thermotropic liquid crystalline conjugated polymer (LCCP) chain orientation. Two recent reports on the coupling of liquid crystal (LC) vibrational modes ${ }^{32}$ and carbon nanotube Wannier excitons ${ }^{33}$ have also shown that $\boldsymbol{\mu . E}$ can be maximized in this way and that polarization-dependence, which has previously been theoretically investigated, ${ }^{34,35}$ allows for applications discussed further in the text.

Previous approaches to LCCP orientation typically used a traditional rubbed polyimide (PI) alignment layer onto which the polymer was spin coated prior to thermal treatment. ${ }^{36}$ The clearing temperatures of LCCPs are relatively high $\left(\sim 200-300^{\circ} \mathrm{C}\right),{ }^{36,37}$ leading to the requirement for a high temperature stable PI, for which there are limited commercial options. Precursor route poly(p-phenylenevinylene) has also been used as an alternative rubbed alignment layer, having the advantage of temperature stability and an electronic structure that more readily permits charge injection from the underlying electrode to the LCCP. ${ }^{38}$ Other approaches to orientation include stretching and rubbing the conjugated polymer, ${ }^{39}$ Langmuir-Blodgett deposition, ${ }^{40}$ use of an aligned host matrix ${ }^{41}$ or nanoimpriting. ${ }^{42}$ Successful fabrication of polarized light emitting diodes ${ }^{38,43,43-46}$ and polarized photoluminescence 
structures ${ }^{30}$ has resulted, together with intrachain mobility enhanced transistors. ${ }^{47}$ For a variety of practical reasons, oriented LCCPs have, however, not been used before in strongly or ultrastrongly-coupled microcavities. The results described within this paper report a new and detailed study of microcavity structures containing films of oriented poly(9,9-dioctylfluorene) (PFO), poly(9,9-dioctylfluorene-co-benzothiadiazole (F8BT) and poly(9,9-dihexylfluoreneco-bithiophene) (F6T2). In addition, instead of using a rubbed PI alignment layer we use here a photoalignment film. Non-contact photoalignment of liquid crystal mesophases has emerged $^{48}$ as a promising alternative to rubbing-induced alignment. Among photoalignment layer materials, the azobenzene-containing Sulfuric Dye 1 (SD1), has shown high temperature stability and remarkable quality for the alignment of low molecular weight LCs. ${ }^{49,50}$ The orientation of F8BT using SD1 has also been recently observed. ${ }^{51}$ Potential advantages of using SD1 photoalignment for photonics and polaritonics are threefold, namely that the SD1 layer can be very thin $(\leq 5 \mathrm{~nm}$ ), that it is almost transparent in the visible (peak absorption at $\sim 3.25 \mathrm{eV}$ ) and that it is a patternable process which allows a straightforward way to fabricate novel photonic structures. ${ }^{50,52}$

We report a detailed study of the use of oriented LCCP films within metallic microcavities. PFO, F8BT and F6T2 films are oriented with SD1 photoalignment layers and in all three cases we demonstrate a systematic enhancement of $\hbar \Omega_{\mathrm{R}_{\mathrm{TE}}}$, for TE-polarized light parallel to the chain orientation direction, compared to non-aligned reference samples. The maximum $\hbar \Omega_{\mathrm{R}_{\mathrm{TE}}}=1.80 \pm 0.01 \mathrm{eV}(689 \mathrm{~nm})$ is for $\mathrm{F} 6 \mathrm{~T} 2$, a value that would sit within the visible spectrum. This structure also gives the largest normalized coupling ratio, $g=65 \%$, reported to date for an organic semiconductor microcavity. Photoluminescence for TE (parallel to the alignment direction) and corresponding TM polarizations makes the changes in coupling strength between polarizations evident for all three polymers. Going beyond enhancement of the Rabi-splitting energy, we discuss the potential use of organic microcavities containing uniaxially oriented LCCP films for demonstration of the elusive polaritonic NOT gate, ${ }^{53-55}$ for quantum simulation through complex energy landscapes and more generally 
its advantages for the realization of polarization sensitive devices, lasing and condensation related phenomena.

\section{Results And Discussion}

The optical constants for thin films of PFO, F8BT, F6T2 (see Methods for all fabrication protocols) were extracted using Variable Angle Spectroscopic Ellipsometry (VASE). As the

polymer chains tend to lie in the plane of the film, ${ }^{56-58}$ the resulting optical constants are well fitted ${ }^{27,59}$ using an in-plane/out-of plane anisotropic model yielding the components $n_{\text {ord }}, k_{\text {ord }}, n_{\text {ex }}, k_{\text {ex }}$ of the complex refractive index $\tilde{n}=n+i k$. Figure 1 shows (in green) the in-plane optical constants $\left(n_{\text {ord }}, k_{\text {ord }}\right)$ for F8BT (b), PFO (c) and F6T2 (d) (The complete sets of optical components extracted are available in Supplementary Information). All spectra comprise either one or several inhomogeneously broadened distributions of chain-delocalised exciton transitions $\left(E_{\mathrm{X}_{\mathrm{PFO}}}\right.$ peaked at around $3.23 \mathrm{eV}, E_{\mathrm{X}_{\mathrm{F}_{8 \mathrm{BT}}}}$ and $E_{\mathrm{X}_{\mathrm{F}_{8 \mathrm{BT}}}}$ peaked at respectively $3.82 \mathrm{eV}$ and $2.70 \mathrm{eV}$ and $E_{X_{F 6 T 2}}$ at $2.79 \mathrm{eV}$ ), with higher lying features above $5 \mathrm{eV}$ associated to ring-localized fluorene states. ${ }^{60}$

Following photoalignment of an SD1 spincoated film in the in-plane $y$ direction, the optical components $n_{x}, n_{y}, n_{z}, k_{x}, k_{y}, k_{z}$ were extracted using a biaxial anisotropic model ${ }^{61}$ and are shown in Figure 1(a). As expected, the alignment brings about intense $n_{y}, k_{y}$ optical components compared to their perpendicular counterparts $n_{x}, k_{x}$. Polymer layers of F8BT, PFO and F6T2 were then spincoated on top of the photoaligned SD1, thermally annealed into their respective nematic phases $\left(250^{\circ} \mathrm{C}\right.$ for $\mathrm{F} 8 \mathrm{BT}, 160^{\circ} \mathrm{C}$ for $\mathrm{PFO}$ and $220^{\circ} \mathrm{C}$ for $\left.\mathrm{F} 6 \mathrm{~T} 2\right)$ and subsequently quenched to room-temperature. Homogeneous (in-plane) uniaxial alignment is thereby generated along the $y$ direction, with quenching freezing the order within a nematic glass state. The corresponding optical components of the obtained films were fitted and are shown in Figure 1(b),(c),(d) with $n_{y}, k_{y}$ substantially larger than $n_{x}, k_{x}$. The $x$ axis matches the direction of the polarizer used during the photoalignment of SD1 which confirms that 
the polymer chains align parallel to SD1, orthogonal to the electric field direction $x$ to which the SD1 film was exposed. ${ }^{49,50}$ The use of the $(x, y)$ coordinate system is further validated in Supporting Information by measuring the s- and p- polarized transmittances at normal incidence while rotating a thin film of oriented PFO.
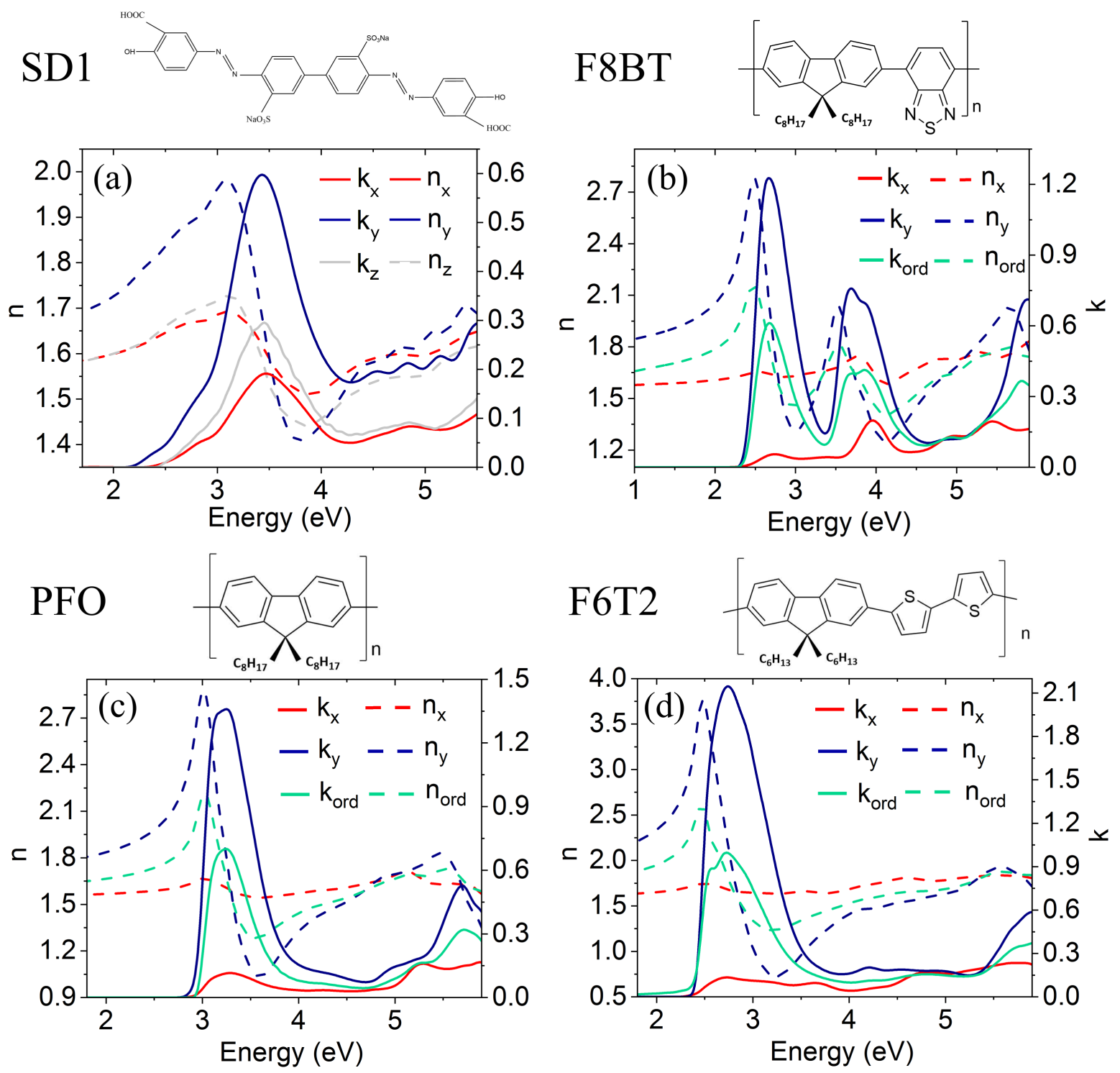

Figure 1: (a) Optical components $n_{x} n_{y}, n_{z}, k_{x}, k_{y}, k_{z}$ for a thin film of SD1 aligned along the y direction. (b),(c),(d) In green: In-plane $n_{\text {ord }}, k_{\text {ord }}$ optical components for spincoated F8BT (b), PFO (c) and F6T2 (d). The in-plane optical components of the films following alignment are shown in blue for $n_{y}, k_{y}$ (parallel to the alignment direction) and in red for $n_{x}, k_{x}$ (perpendicular to the alignment direction) for SD1 (a), F8BT (b), PFO (c) and F6T2 (d). Dashed lines give the real component of the complex refractive index $\tilde{n}=n+i k$, solid lines the imaginary component. 
Time-integrated photoluminescence (PL) spectra were recorded at normal incidence for the three polymers and are shown in Figure 2 (see Methods for the experiment geometry). The spectra were measured at normal incidence with the collection polarizer both in vertical (blue) and horizontal (red) positions; for the aligned films, the vertical direction matched the direction of the alignment. In each case, we calculate the integrated ratio $R_{\mathrm{VH}}=\int \frac{I_{\mathrm{V}}(E)}{I_{\mathrm{H}}(E)} d E$ which reveals the presence of in-plane uniaxial alignment in the film. For F8BT, the spectrum of the non-aligned film (Figure 2 (a)) reveals an inhomogeneously broadened distribution with $S_{1}-S_{0}(0-0),(0-1)$ vibronic peaks located at $2.29 \mathrm{eV}(541 \mathrm{~nm})$ and $2.15 \mathrm{eV}(577 \mathrm{~nm})$. We calculate $R_{\mathrm{VH}}=1.08$, with the deviation from unity being fully accounted for by the degree of polarization of the excitation laser beam; as expected the polymer chains have no preferential in-plane orientation. For the aligned F8BT (Figure 2 (b)), the same spectral positions for the vibronic peaks are recorded but with a difference in relative heights due to thickness variations between the aligned and non-aligned films. The integrated ratio $R_{\mathrm{VH}}=8.3$ confirms a significant in-plane preferential alignment of polymer chains within the emitting layer (in-depth studies relating the polarized emission to the microscopic parameters of aligned F8BT can be found in Ref 44). For non-aligned PFO (Figure 2(c)), the $S_{1}-S_{0}$ well resolved (0-0), (0-1) and (0-2) PL vibronic peaks appear at $2.88 \mathrm{eV}(430 \mathrm{~nm}), 2.71 \mathrm{eV}$ $(457 \mathrm{~nm})$ and $2.58 \mathrm{eV}(481 \mathrm{~nm})$ with an integrated ratio $R_{\mathrm{VH}}$ that increases from 1.09 to 6.9 from non-aligned to aligned film (Figure 2(d)). As the vibronic structure is well resolved in this case, we calculate the peak ratios $R_{\mathrm{pVH}}(E)=\frac{I_{\mathrm{V}}(E)}{I_{\mathrm{H}}(E)}$ for $R_{\mathrm{pVH}}(2.88), R_{\mathrm{pVH}}(2.71)$ and $R_{\mathrm{pVH}}(2.58)$, yielding ratios $11.2,9.5$ and 8.2 , respectively. These results are comparable to those obtained previously using rubbed PI layers. ${ }^{43}$ For non-aligned F6T2 (Figure 2(e)), the PL vibronic peaks appear at $2.28 \mathrm{eV}(544 \mathrm{~nm}), 2.12 \mathrm{eV}(585 \mathrm{~nm})$ and $1.95 \mathrm{eV}(636 \mathrm{~nm})$ and $R_{\mathrm{VH}}$ increases from a value of 1.16 to 3.50 (Figure 2(f)), albeit for a ratio that is harder to accurately determine than for F8BT and PFO, due to weaker light emission and an enhanced noise floor that needed to be removed using a fast Fourier transform algorithm. 

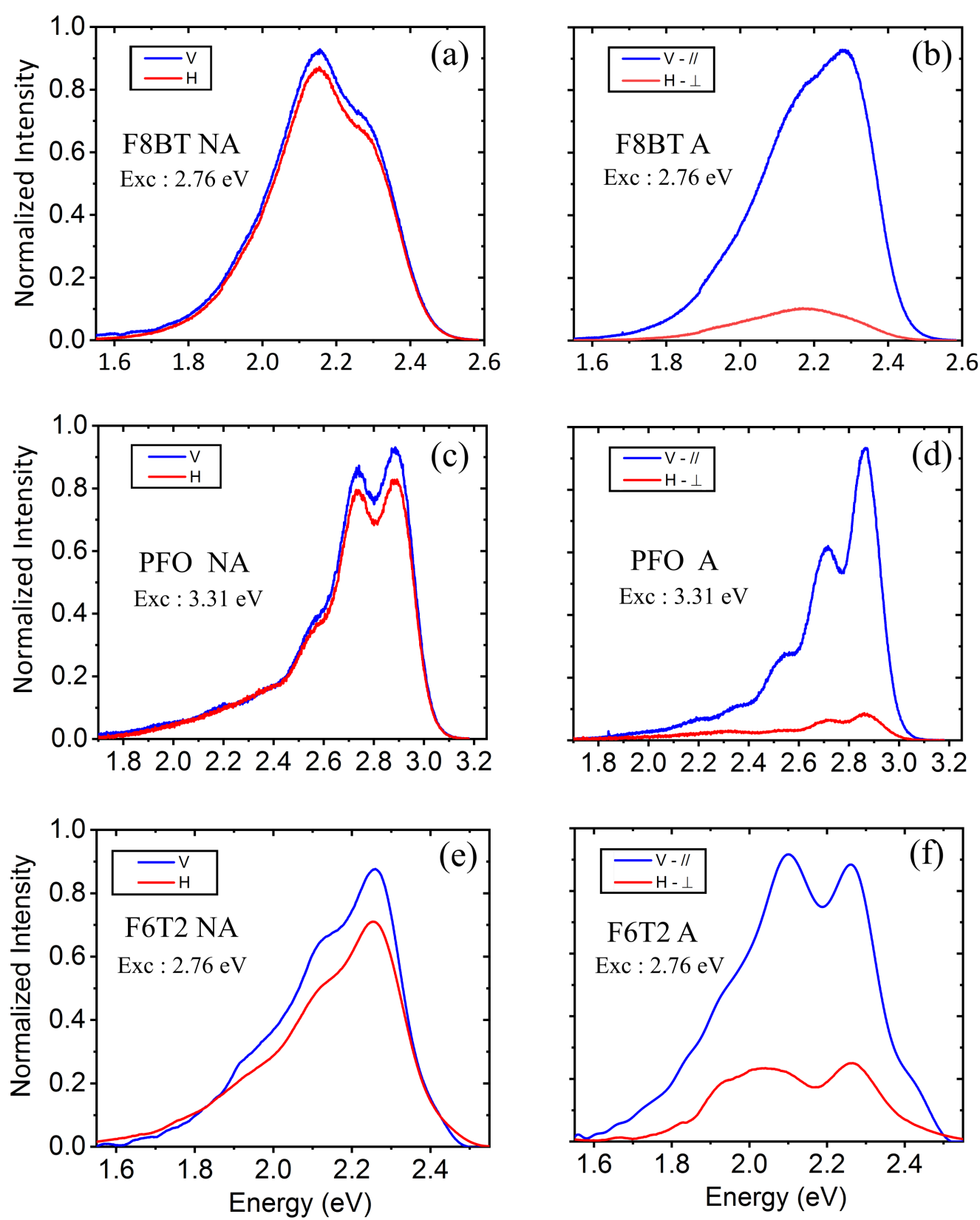

Figure 2: PL spectra for non-aligned ((a),(c) \& (e)) and aligned ((b),(d) \& (f)) F8BT ((a) \& (b)), PFO ((c) \& (d)) and F6T2 ((e) \& (f)) thin films. For a given polymer in a given alignment state, the parallel and perpendicular spectra were plotted on the same scaled intensity axis range to allow for their comparisons. Solid blue lines show the PL collected using a polarizer in the vertical direction and red lines in the horizontal direction. In panels (b), (d) and (f): // and $\perp$ indicate that the collection polarizer direction respectively matches or is perpendicular to the orientation direction of the polymer chains in the film. A fast Fourier transform algorithm was used for F6T2 ((e) \& (f)) to reduce noise in the recorded signal. 
Al-Polymer-Al microcavities were fabricated using both non-aligned and aligned F8BT, PFO and F6T2 polymer films with chain orientation achieved via SD1 photoalignment. Angle-resolved polarized reflectivity maps were recorded for each microcavity by varying the angle $\theta$ formed between the vector normal to the microcavity plane and the incident light direction. For the oriented polymer cavities, the measurement was performed at an angle $\Phi$ (formed between TE polarization and the polymer chain direction) equal to $0^{\circ}$. All results were analysed using a Hopfield-Agranovich Hamiltonian ${ }^{22,62,63}$ including either one (PFO, F6T2) or two (F8BT) separate excitons, with all fitting results displayed in Table 1. For each microcavity, the experimental results are supported by transfer matrix reflectivity (TMR) calculations whose outputs are shown in Supporting Information. The reference results obtained for the non-aligned PFO and F8BT cavities are also shown in Supporting Information and confirm previous reports ${ }^{27,59}$ in which $\hbar \Omega \sim 1 \mathrm{eV}$ has been observed for glassy PFO.

Measured and fitted results for the non-aligned F6T2 cavity for TE polarization are shown in Figure 3 (a) where we observe the Lower (LP) and Upper (UP) Polariton branches split about the exciton energy $\left(E_{\mathrm{X}_{\mathrm{F} 6 \mathrm{~T} 2}}=2.72 \mathrm{eV}\right)$, with a separation of more than $1 \mathrm{eV}$ that provides clear evidence of USC. Even though the layer is non-aligned, the measured Rabi splitting energy $\hbar \Omega_{0_{\mathrm{TE}}}=1.38 \pm 0.01 \mathrm{eV}$, corresponding to a coupling ratio $\mathrm{g}=49 \%$, is larger than previously reported splitting values. As expected, the results for TM polarization (Figure 4 (a)) display a flatter angular dispersion for the polaritons as the effective refractive index $n_{\mathrm{eff}_{\mathrm{TM}}}$ of the microcavity increases due to polarization-dependent penetration through the metallic mirrors. ${ }^{10,64,65}$ For this polarization, the in-plane/out-of-plane anisotropy of the active layer slightly reduces the Rabi energy down to $\hbar \Omega_{0_{\mathrm{TM}}}=1.27 \pm 0.01 \mathrm{eV}$, as the contribution of the weaker out-of plane $\left(k_{\mathrm{ex}}\right)$ component to the overall interaction increases along with $\theta \cdot{ }^{10}$

Results for the aligned PFO, F6T2 and F8BT cavities are shown, respectively, in Figure 3 (b), (c) and (d) for TE polarization at $\Phi=0^{\circ}$. In each case, USC is clearly observed with 
fitting results revealing a systematic increase for $\hbar \Omega_{0_{\mathrm{TE}, \Phi=0^{\circ}}}$ of $\sim 40 \%$ compared to the nonaligned cavities. As the transition dipole moment $\boldsymbol{\mu}$ rotates together with the polymer chains in the $y$-direction, the relative increase in coupling strength can be calculated using the term $\boldsymbol{\mu} . \boldsymbol{E}$ in Equation 1 to be: $\frac{\cos (0)}{\cos \left(\frac{\pi}{4}\right)}-1=(\sqrt{2}-1) \sim 41 \%$ for TE polarization.

All the $\hbar \Omega_{0_{\mathrm{TE}, \Phi=0^{\circ}}}$ values derived here, apart from that just reported for non-aligned F6T2, exceed previous reports: the aligned F8BT cavity exhibits $\hbar \Omega_{01_{\mathrm{TE}, \Phi=0^{\circ}}}=1.18 \pm 0.01 \mathrm{eV}$ and $\hbar \Omega_{02_{\mathrm{TE}, \Phi=0^{\circ}}}=1.25 \pm 0.01 \mathrm{eV}$, the aligned PFO cavity $\hbar \Omega_{0_{\mathrm{TE}, \Phi=0^{\circ}}}=1.47 \pm 0.01 \mathrm{eV}$ and the aligned F6T2 cavity reaches an unprecedented value of $\hbar \Omega_{0_{\mathrm{TE}, \Phi=0^{\circ}}}=1.80 \pm 0.01 \mathrm{eV}$. To our knowledge, this last value is the first Rabi splitting for a solid-state system to reach a value comparable to photons in the visible spectrum $(\sim 689 \mathrm{~nm})$, exceeding by more than $60 \%$ the previous record. ${ }^{14,15}$ The corresponding coupling ratio $g \sim 65 \%$ is also slightly higher than that reported in Ref 25, a notable result given that the $g$-value reported here was obtained for an exciton lying some $\sim 1.6 \mathrm{eV}$ higher in energy. 


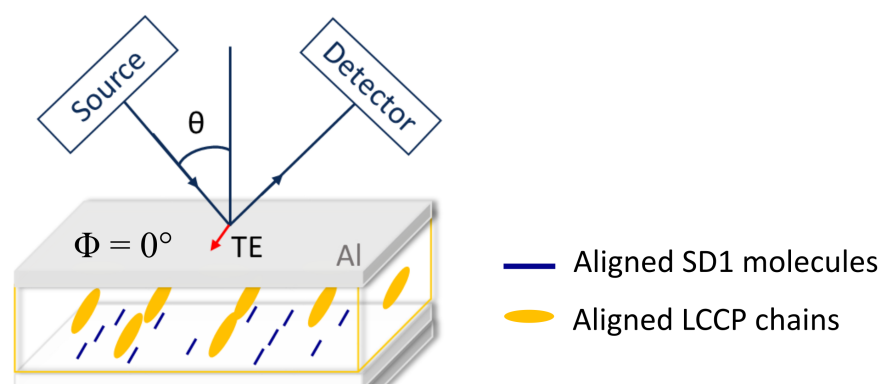

(a) $\quad$ F6T2 NA

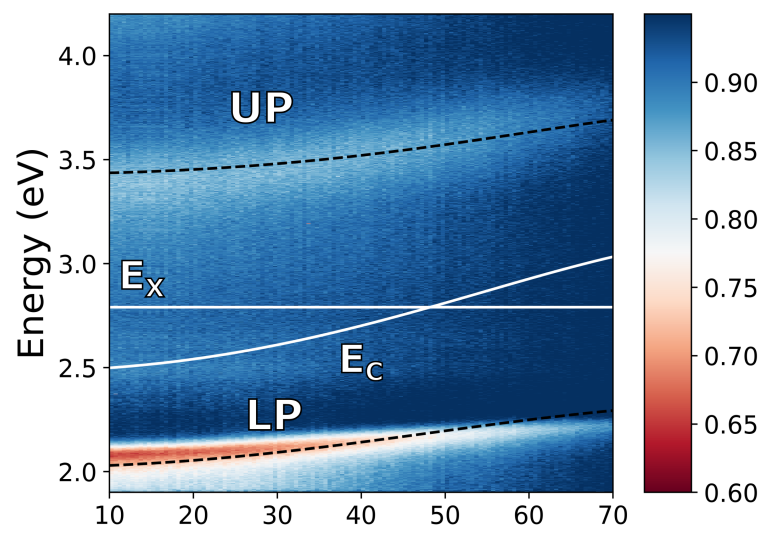

(c) $\quad$ F6T2 A $-\Phi=0^{\circ}$

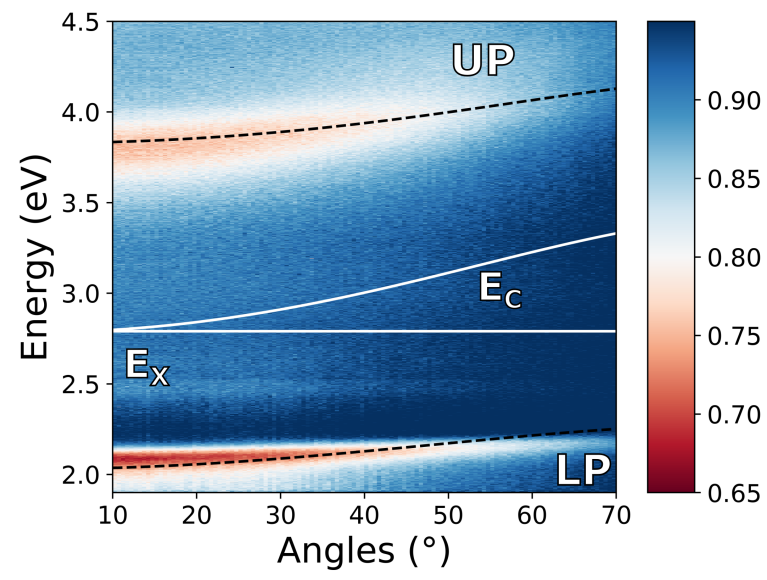

(b) $\quad$ PFO A $-\Phi=0^{\circ}$

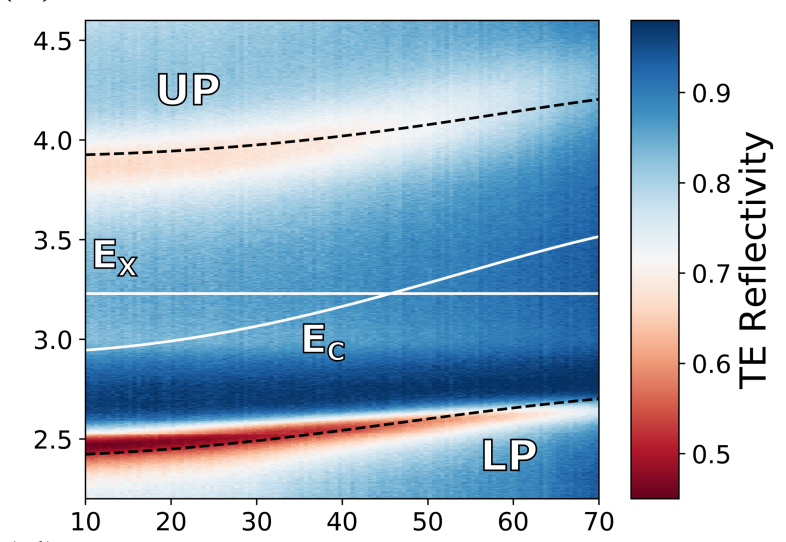

(d) $\quad$ F8BT A $-\Phi=0^{\circ}$

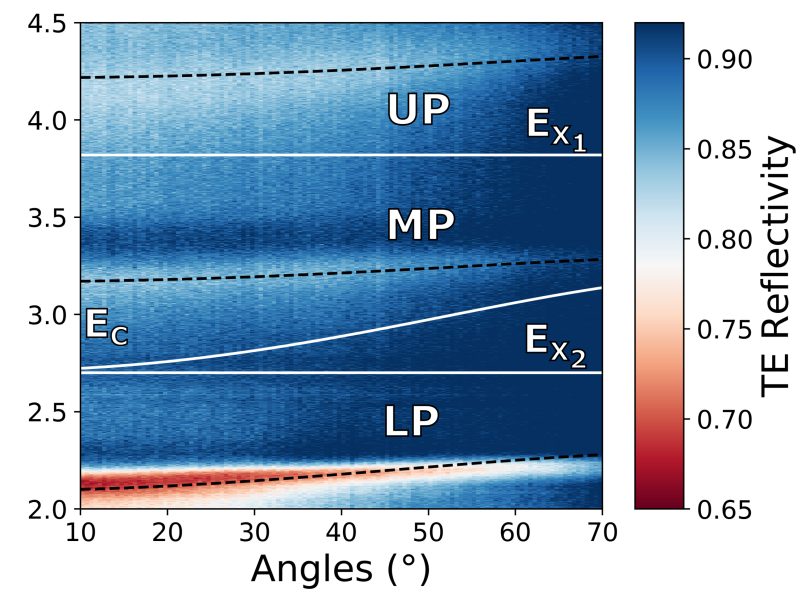

Figure 3: Experimental, angle-resolved, TE-polarized reflectivity maps for microcavities containing: (a) non-aligned F6T2, (b) aligned PFO, (c) aligned F6T2 and (d) aligned F8BT. For (b), (c) and (d), the measurements were performed at $\Phi=0^{\circ}$ (see text and schematic experimental geometry shown above the maps). The overlaid solid white lines show the exciton $E_{\mathrm{X}}$ and cavity mode $E_{\mathrm{C}}$ dispersions and the black dashed lines the polariton branch dispersions derived from the analytical model. Note the Fano-like asymmetry around the LP for (a), (b) and (c) similar to that observed in Ref 10 and the reduced LP linewidth relative to the UP as a consequence of motional narrowing. ${ }^{66,67}$ 
The results for TM polarization for the aligned polymer cavities are shown in Figure 4 (b), (c) and (d). In those measurements, the weak coupling regime is clearly observed for F6T2 (c) and F8BT (d) as the LP and UP are no longer visible and are replaced by a single photonic mode. For PFO (b), the reflectivity observed does not show proper anti-crossing around the exciton $\left(E_{\mathrm{X}_{\mathrm{PFO}}}=3.23 \mathrm{eV}\right)$, with PL measurements in the next section (vide infra) further showing a lack of evidence for SC. These results offer exciting new opportunities that are further examined in the discussion section below. 
(a)

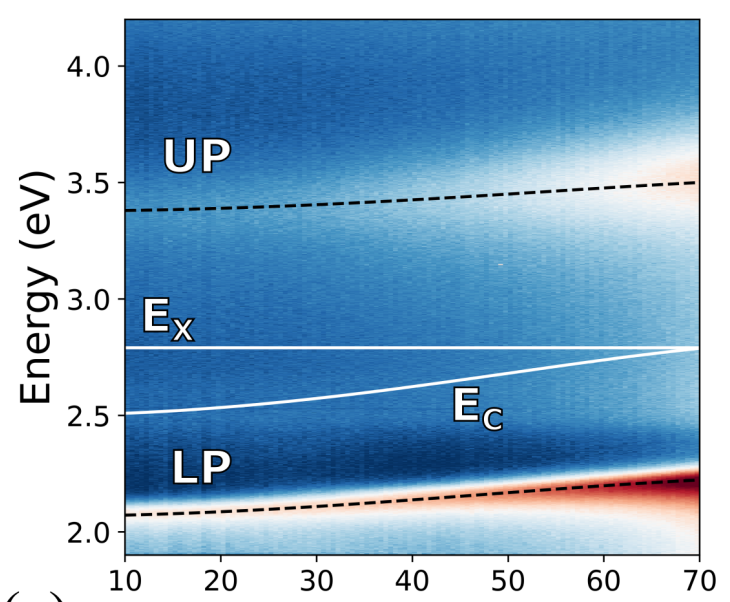

(c)

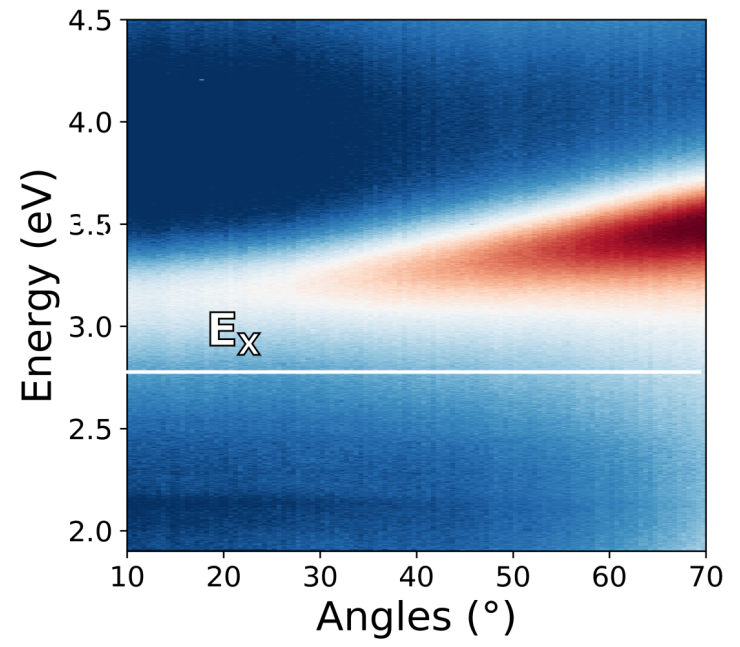

(b) $\quad$ PFO A - $\Phi=0^{\circ}$

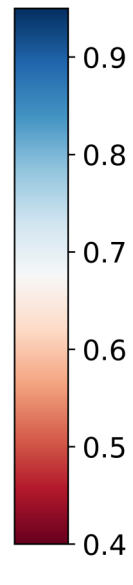

(d)

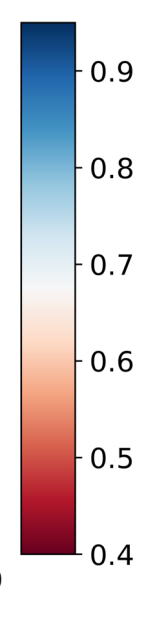

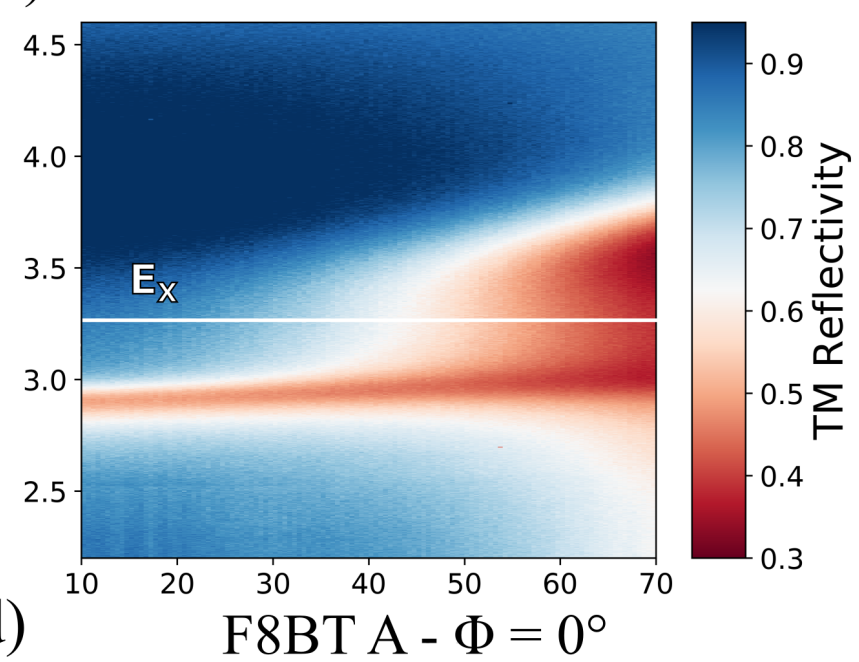

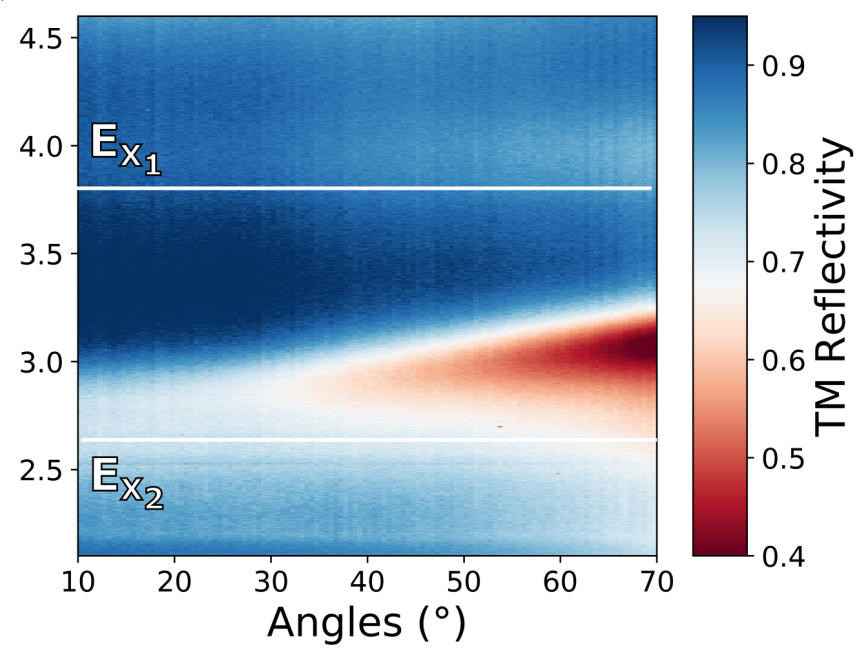

Figure 4: Experimental, angle-resolved, TM-polarized reflectivity maps for microcavities containing (a) non-aligned F6T2, (b) aligned PFO, (c) aligned F6T2 and (d) aligned F8BT. For (b), (c) \& (d), the measurements were performed at $\Phi=0^{\circ}$ (see text for definition) and the overlaid solid white lines show the respective exciton energies, $E_{\mathrm{X}}$. In (a) in addition to $E_{\mathrm{X}}$, the second white line presents the cavity mode dispersion, $E_{\mathrm{C}}$. The black dashed lines show the polariton branch dispersions derived from the analytical model. 
Table 1: Extracted and pre-set parameter values for microcavities containing non-aligned and aligned polymers, modelled using a Hopfield-Agranovich Hamiltonian. ${ }^{22,62,63}$ Values are shown for both TE- and TM-polarization, at $\Phi=0^{\circ}$ (see definition in the text) for the aligned cavities. The initials $\mathrm{A}$ and NA respectively designate aligned and non-aligned polymer layers.

\begin{tabular}{|c|c|c|c|c|c|c|}
\hline Polymer: & PFO NA & PFO A & F8BT NA & F8BT A & F6T2 NA & F6T2 A \\
\hline$\hbar \omega_{1}(\mathrm{eV})^{1}$ & 3.23 & 3.23 & 3.82 & 3.82 & 2.79 & 2.79 \\
\hline$\hbar \omega_{2}(\mathrm{eV})^{2}$ & - & - & 2.70 & 2.70 & - & - \\
\hline$\hbar \Omega_{01_{\mathrm{TE}}}(\mathrm{eV})^{3}$ & $1.02 \pm 0.01$ & $1.47 \pm 0.01$ & $0.84 \pm 0.01$ & $1.18 \pm 0.01$ & $1.38 \pm 0.01$ & $1.80 \pm 0.01$ \\
\hline$g_{01_{\mathrm{TE}}} \%^{4}$ & 32 & 46 & 22 & 31 & 49 & 65 \\
\hline$\hbar \Omega_{01_{\mathrm{TM}}}(\mathrm{eV})^{5}$ & $0.97 \pm 0.01$ & - & $0.80 \pm 0.01$ & - & $1.27 \pm 0.01$ & - \\
\hline$g_{01_{\mathrm{TM}}} \%^{6}$ & 30 & - & 21 & - & 46 & - \\
\hline$\hbar \Omega_{02_{\mathrm{TE}}}(\mathrm{eV})^{7}$ & - & - & $0.84 \pm 0.01$ & $1.25 \pm 0.01$ & - & - \\
\hline$g_{02 \mathrm{TE}} \%^{8}$ & - & - & 31 & 46 & - & - \\
\hline$\hbar \Omega_{02_{\mathrm{TM}}}(\mathrm{meV})^{9}$ & - & - & $0.80 \pm 0.01$ & - & - & - \\
\hline$g_{02_{\mathrm{TM}}} \%^{10}$ & - & - & 30 & - & - & - \\
\hline$n_{\mathrm{eff}_{\mathrm{TE}}} 11$ & $1.68 \pm 0.01$ & $1.70 \pm 0.01$ & $1.70 \pm 0.01$ & $1.87 \pm 0.01$ & $1.64 \pm 0.01$ & $1.71 \pm 0.01$ \\
\hline$n_{\mathrm{eff}_{\mathrm{TM}}}{ }^{12}$ & $2.33 \pm 0.01$ & - & $2.37 \pm 0.01$ & - & $2.12 \pm 0.01$ & - \\
\hline$E_{\mathrm{c}_{\mathrm{TE}}}(0)(\mathrm{eV})^{13}$ & $3.13 \pm 0.02$ & $2.92 \pm 0.02$ & $2.54 \pm 0.02$ & $2.71 \pm 0.02$ & $2.48 \pm 0.02$ & $2.78 \pm 0.02$ \\
\hline$E_{\mathrm{c}_{\mathrm{TM}}}(0)(\mathrm{eV})^{14}$ & $3.15 \pm 0.02$ & _ & $2.59 \pm 0.02$ & - & $2.50 \pm 0.02$ & - \\
\hline
\end{tabular}

${ }^{1}$ Exciton oscillator 1 transition energy.

${ }^{2}$ Exciton oscillator 2 transition energy.

${ }^{3}$ TE-polarized Rabi energy associated with exciton 1 for $\omega_{\mathrm{Cav}_{\mathrm{TE}}}=\omega_{1}$ (see definition in the text).

${ }^{4}$ TE-polarized normalized coupling ratio energy associated with exciton 1 (see definition in the text).

${ }^{5}$ TM-polarized Rabi energy associated with exciton 1 for $\omega_{\mathrm{Cav}_{\mathrm{TM}}}=\omega_{1}$ (see definition in the text).

${ }^{6}$ TM-polarized normalized coupling ratio energy associated with exciton 1 (see definition in the text).

7 TE-polarized Rabi energy associated with exciton 2 for $\omega_{\mathrm{cav}}{ }_{\mathrm{TE}}=\omega_{2}$ (see definition in the text).

8 TE-polarized normalized coupling ratio energy associated with exciton 2 (see definition in the text).

${ }^{9}$ TM-polarized Rabi energy associated with exciton 2 for $\omega_{\mathrm{Cav}_{\mathrm{TM}}}=\omega_{2}$ (see definition in the text).

${ }^{10}$ TM-polarized normalized coupling ratio energy associated with exciton 2 (see definition in the text).

${ }^{11}$ Effective refractive index for TE polarization.

${ }^{12}$ Effective refractive index for TM polarization.

${ }^{13}$ TE-polarized energy of the bare cavity mode at normal incidence.

${ }^{14}$ TM-polarized energy of the bare cavity mode at normal incidence. 
Angle-resolved PL was recorded for each microcavity using the same laser source and excitation geometry as used for the thin film PL measurements; the laser beam was focused onto the sample at a fixed angle of incidence of $75^{\circ}$. For each microcavity, the average laser power was kept low $(\leq 10 \mu \mathrm{W})$ and the photon energy was chosen to optically pump one of the intense absorptions arising from the optical transitions of the underlying polymer. For the aligned cavities, the measurement was performed at $\Phi=0^{\circ}$.

Figure 5 shows PL intensity maps plotted on an energy vs emission angle for both TE((a), (c) and (e))) and TM-polarized ((b), (d) and (f)) light emission from the non-aligned F8BT ((a) and (b)), PFO ((c) and (d)) and F6T2 ((e) and (f)) microcavities. In each case the emission is dominated by a narrow single peak originating from the LP which is relatively insensitive to angular-dispersion: a recognizable feature of USC. ${ }^{10-12}$ Table 2 reports the peak positions and full width at half maximum (FWHM) values at normal incidence, together with the angular dispersion from 0 to $60^{\circ}$. The results for PFO agree with a previous report ${ }^{27}$ and the range of polymers used here allows for emissions across a large part of the visible spectrum; in the blue at $\sim 452 \mathrm{~nm}$ for $\mathrm{PFO}$, green at $\sim 537 \mathrm{~nm}$ for F8BT and yellow/orange at $\sim 588 \mathrm{~nm}$ for F6T2.

Corresponding PL intensity maps for the aligned cavities are shown in Figure 6. TEpolarized emission ((a), (c) and (f)) resembles that observed from non-aligned microcavities with a single LP peak. There is however an energy shift for F8BT and PFO between nonaligned and aligned cavities: the peak emission is recorded at $2.13 \mathrm{eV}$ at normal incidence for aligned F8BT compared to $2.31 \mathrm{eV}$ when non-aligned, and at $2.59 \mathrm{eV}$ for aligned $\mathrm{PFO}$ compared to $2.74 \mathrm{eV}$ when non-aligned. These redshifts are not the result of different thicknesses between the cavities as TMR calculations show that for each pair, the polymer layer thicknesses are comparable (the aligned F8BT is $110 \mathrm{~nm}$-thick compared to $118 \mathrm{~nm}$ when non-aligned, the aligned PFO $97 \mathrm{~nm}$-thick compared to $96 \mathrm{~nm}$ when non-aligned). They are, rather, direct evidence of the increased interaction strength which repels the UP and LP to respectively higher and lower energies. The emission for aligned and non-aligned 
F6T2 is closer in energy at normal incidence $(2.11 \mathrm{eV}$ aligned compared with $2.10 \mathrm{eV}$ nonaligned) and is this time the result of a much larger thickness for the non-aligned F6T2 layer; the aligned F6T2 is $94 \mathrm{~nm}$-thick compared to $123 \mathrm{~nm}$ non-aligned film. This results in lower cavity mode and LP energies in the non-aligned case, as observed in Figure 3, which compensates for the difference in interaction strength between the two microcavities.

TM-polarized measurements ((b), (d) and (f)) show no distinguishable emission for F8BT (b) and F6T2 (g) as the only photonic mode in both those cavities lies too high in energy to allow any emission from the underlying polymer. The cavity modes are at $\sim 3 \mathrm{eV}$ for F6T2 and $\sim 2.9 \mathrm{eV}$ for F8BT whereas PL emission is located at energies lower than $2.6 \mathrm{eV}$ for both F6T2 and F8BT (Figure 2 (b) and (f)). For the microcavity containing aligned PFO (d), a 5-times weaker, broad, angle-independent TM-polarized emission was detected, with two maxima at 2.88 and $2.71 \mathrm{eV}$ coinciding with the PL peaks from the bare film (Figure 2(d)). This confirms that the microcavity no longer operates under USC for this polarization. The emission emerges via the photonic mode at $\sim 2.88 \mathrm{eV}$ (see 2(b)) and even though this photonic mode overlaps with the most intense part of the bare film PL, the resulting signal is much weaker in TM than TE polarization as the emission in the direction perpendicular to the chain orientation direction is much weaker. 

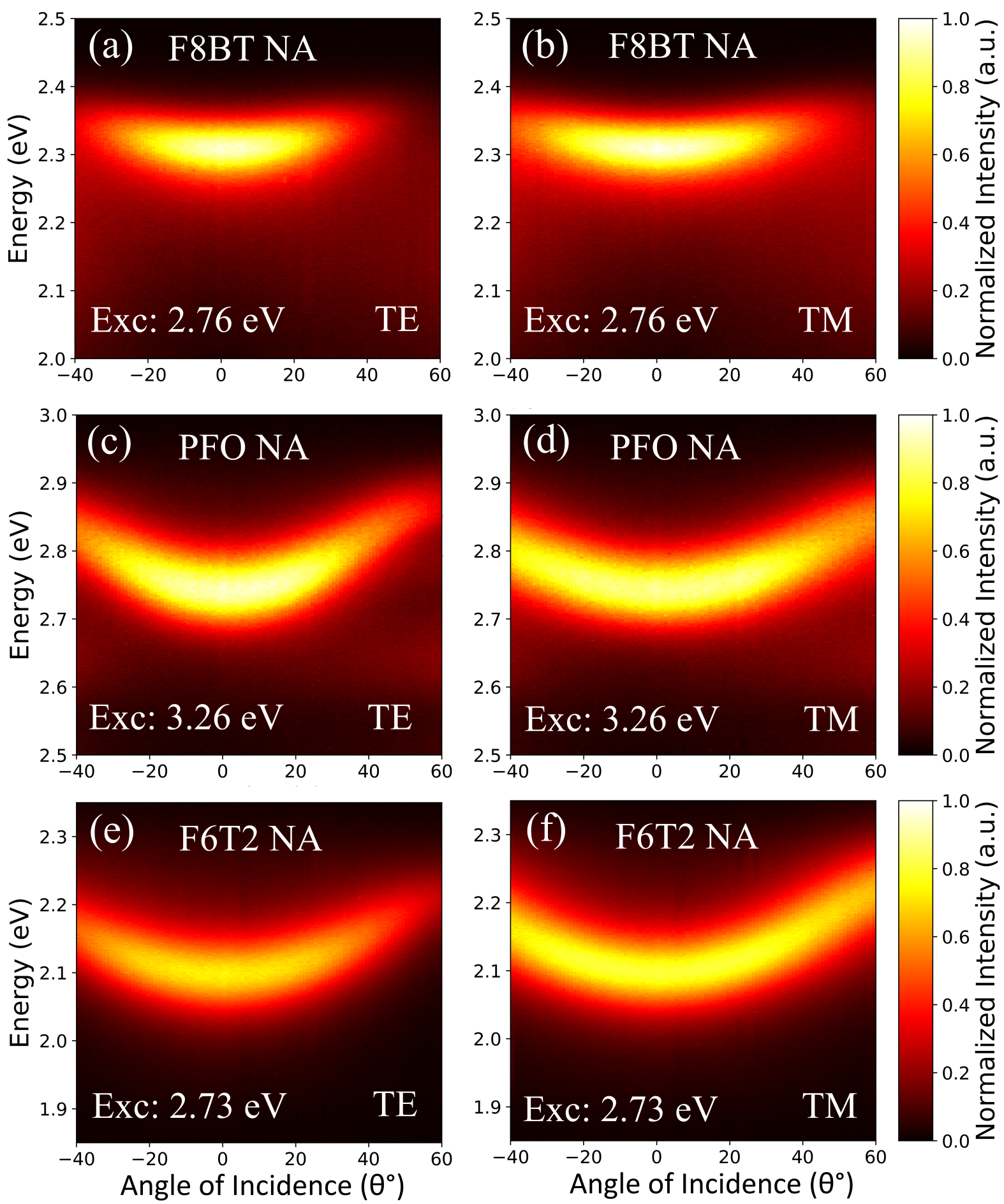

Figure 5: Angle-resolved PL spectral intensity maps for microcavities containing non-aligned F8BT ((a) \& (b)), PFO ((c) \& (d)) and F6T2 ((e) \& (f)), with TE ((a), (c), (e)) and TM $((\mathrm{b}),(\mathrm{d}),(\mathrm{f}))$ polarized spectra plotted separately. The excitation energy, Exc, is given for each measurement. Each polarization pair ((a) \& (b)), ((c) \& (d)), ((e) \& (f)) was plotted on the same scaled intensity axis range. 

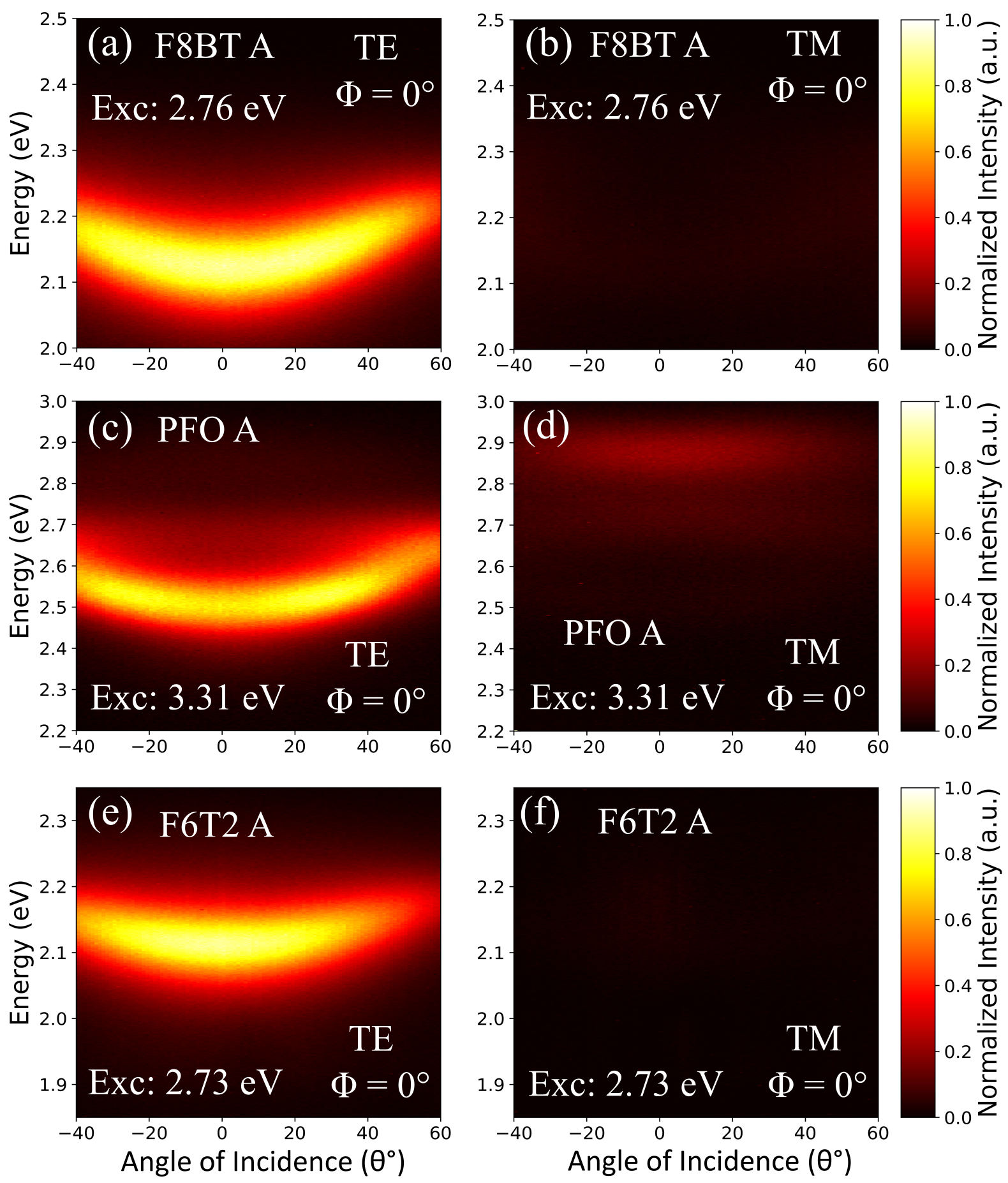

Figure 6: Angle-resolved PL spectral intensity maps for microcavities containing aligned F8BT ((a) \& (b)), PFO ((c) \& (d)) and F6T2 ((e) \& (f)), with TE ((a), (c), (e)) and TM ((b), (d), (f)) polarized spectra plotted separately. The measurements were performed at $\Phi=0^{\circ}$ (see text for definition). Each polarization pair ((a) \& (b)), ((c) \& (d)), ((e) \& (f)) was plotted on the same scaled intensity axis range. 
Table 2: Peak positions and FWHM values at normal incidence $\left(\theta=0^{\circ}\right)$ for the TE- and TM-polarized emission s displayed in Figures 5 and 6 . The angular dispersion of the emission for both polarizations from $\theta=0$ to $60^{\circ}$ is also reported. The initials A and NA respectively designate aligned and non-aligned polymer layers.

\begin{tabular}{c|c|c|c|c|c|c} 
Polymer: & PFO NA & PFO A & F8BT NA & F8BT A & F6T2 NA & F6T2 A \\
Peak position (eV) & 2.74 & 2.59 & 2.31 & 2.13 & 2.10 & 2.11 \\
FWHM $_{\mathrm{TE}}(\mathrm{meV})$ & $99 \pm 1$ & $140 \pm 5$ & $89 \pm 5$ & $127 \pm 1$ & $109 \pm 1$ & $109 \pm 1$ \\
FWHM $_{\mathrm{TM}}(\mathrm{meV})$ & $99 \pm 1$ & - & $82 \pm 5$ & - & $109 \pm 1$ & - \\
TE dispersion (meV) & 130 & 50 & 30 & 80 & 110 & 60 \\
TM dispersion (meV) & 110 & - & 20 & - & 110 & -
\end{tabular}

Several unique opportunities exist for anisotropic polaritons: chain orientation could enable table-top observation of topologically protected edge modes in plexcitonic systems, ${ }^{68}$ the possibility to align SD1 at mesoscopic scales ${ }^{50-52}$ allows for a local orientation of the polymer chains that could lead to fabrication of the missing NOT gate in polaritonic optical logic thanks to a spontaneous splitting between TE and TM polarizations. ${ }^{53-55}$ It should also enable the fabrication of complex energy landscapes, especially when combined with tunability of molecular conformation, for example by generating segments of $\beta$-phase in $\mathrm{PFO}^{69}$ and copolymers. ${ }^{70}$ This could help address challenges in quantum simulation. ${ }^{71}$ We further expect that a wide range of polarization sensitive devices (photodiodes, ${ }^{72}$ transistors ${ }^{7}$ ) and phenomena, including Bose-Einstein condensation and exciton-polariton lasing (recently demonstrated with pentafluorene ${ }^{73}$ and $\mathrm{PFO}^{74}$ ) will take advantage of the polarizationdependent coupling; the spontaneous polarization observed during lasing and condensation could for instance be controlled by molecular orientation.

\section{Conclusions}

We have fabricated organic microcavities containing LCCPs PFO, F8BT and F6T2, aligned using thin SD1 photoalignment layers. Microcavities with non-aligned films yield USC with $\hbar \Omega_{\mathrm{R}_{\mathrm{F} 6 \mathrm{~T} 2}}=1.34 \mathrm{eV}$ for $\mathrm{F} 6 \mathrm{~T} 2$, a value that exceeds previous reports. Alignment subsequently 
allowed a systematic increase in coupling strength for light polarised parallel to the polymer chain direction, with $\hbar \Omega_{\mathrm{R}}$ reaching as high as $1.80 \mathrm{eV}$, again for F6T2; an unprecedented value comparable to photon energies in the red part of the visible spectrum. This value further leads to the highest coupling ratio $g=65 \%$, reported to date for a solid-state system. ${ }^{16,17}$ TE-polarized, angle-resolved PL, with excitation $\boldsymbol{E}$-vector parallel to the chain orientation direction, revealed LP emission red-shifted relative to that from the non-aligned cavities, a clear signature of the increased interaction strength. In this geometry, the absence of polariton branches from the TM-polarized reflectivity together with weak or absent PL emission confirmed polarization dependent coupling strengths. Finally, by demonstrating this behaviour for three different polymers, we confirmed its generality for LCCPs. The opportunity to reach coupling ratios closer to $90 \%$ exists if such alignment can be achieved for LCCPs with excitons at lower energies. Aligning LCCPs using SD1 to achieve microscopic spatial control also offers the possibility to realize key polaritonic devices and rich energy landscapes.

\section{Methods}

\section{Materials}

The three polymers used in this study were supplied by Cambridge Display Technology (F8BT), Sumitomo Chemical (PFO and F6T2) and used as received. Their peak molecular weights were: $M_{p_{\mathrm{PFO}}}=50 \times 10^{3}$ g.mol ${ }^{-1}, M_{p_{\mathrm{F} 8 \mathrm{BT}}}=77 \times 10^{3}$ g.mol ${ }^{-1}, M_{p_{\mathrm{F} 6 \mathrm{~T} 2}}=80 \times 10^{3}$ g. $\mathrm{mol}^{-1}$. The azo-dye photo-alignement layer SD1 was supplied by Dai-Nippon Ink and Chemicals, Japan. Anhydrous toluene (99.8\%), anhydrous chloroform ( $\geq 99 \%)$ and anhydrous 2-methoxyethanol $(\geq 99.8 \%)$ were purchased from Sigma-Aldrich. Solvents were used as received. For the mirror fabrication, Aluminium pellets (99.999\%) were purchased from Kurt J. Lesker. 


\section{Film Fabrication}

The bare films (used for ellipsometry and PL) of SD1, PFO, F8BT, F6T2 were spincoated from solutions in 2-methoxyethanol (SD1 at $1 \mathrm{mg} \cdot \mathrm{mL}^{-1}$ ), toluene (PFO at $18 \mathrm{mg} \cdot \mathrm{mL}^{-1}$ and F8BT at $18 \mathrm{mg} \cdot \mathrm{mL}^{-1}$ ) and chloroform (F6T2 at $\left.13 \mathrm{mg} \cdot \mathrm{mL}^{-1}\right)$. All solutions were prepared in an inert environment, left to stir overnight at a temperature of $55^{\circ} \mathrm{C}$ except for $\mathrm{F} 6 \mathrm{~T} 2$ in chloroform which was left stirring at room temperature. All solutions were then filtered using a $0.45 \mu \mathrm{m}$ PTFE filter. All samples were spincoated on top of fused silica substrates. The non-aligned polymer films were spun for 1 min at a speed of $2000 \mathrm{rpm}$ with an acceleration of $1200 \mathrm{rpm} . \mathrm{s}^{-1}$. For the aligned polymer films, the SD1 layer was first spincoated by spinning $5 \mathrm{~s}$ at $500 \mathrm{rpm}$ (acceleration $500 \mathrm{rpm} . \mathrm{s}^{-1}$ ) and $25 \mathrm{~s}$ at $2000 \mathrm{rpm}$ (acceleration $\left.1200 \mathrm{rpm} . \mathrm{s}^{-1}\right)$. The film was then annealed for 6 minutes at a temperature of $150^{\circ} \mathrm{C}$ to drive any traces of solvent away. The alignment of the SD1 layer was performed in air by exposing the sample to $5 \mathrm{~mW}$ of polarized UV light (emitted by a M365LP1 LED in front of a broadband WP25M-UB polarizer from Thorlabs) for 10 minutes; The SD1 chains aligning perpendicular to the direction of the polarized light. ${ }^{49,50}$ The samples were then put back in an inert environment where the polymer layer was spincoated by spinning for 1 min at a speed of $2000 \mathrm{rpm}$ with an acceleration of $1500 \mathrm{rpm} . \mathrm{s}^{-1}$. Each polymer was then thermally annealed into their respective nematic phases $\left(160^{\circ} \mathrm{C}\right.$ for $\mathrm{PFO}, 220^{\circ} \mathrm{C}$ for $\mathrm{F} 6 \mathrm{~T} 2,250^{\circ} \mathrm{C}$ for F8BT) using a Linkam THMS600 heating stage with a heating rate of $30^{\circ} \mathrm{C} \cdot \mathrm{min}^{-1}$. This temperature was held for 10 minutes and subsequent quenching to room temperature was realized by quickly placing the sample on the metallic floor of the glovebox while applying a gentle flow of nitrogen.

\section{Microcavity Fabrication}

For each microcavity, a $100 \mathrm{~nm}$ thick bottom and approximately $20 \mathrm{~nm}$ thick upper mirror were evaporated at a rate of $10 \mathrm{~A} . \mathrm{s}^{-1}$ at a pressure of $10^{-9} \mathrm{mbar}$. For the non-aligned cavities, the spincoating conditions used on top of the bottom mirror were identical to the 
ones described for the bare films. For the aligned cavities, the SD1 layer was spincoated on top of the bottom mirror. The structures fabricated had low $\mathrm{Q}$ factors $\sim 25$, characteristic of metallic microcavities using Aluminium. The concentration of the SD1 solution in 2methoxyethanol was increased to $3 \mathrm{mg} \cdot \mathrm{mL}^{-1}$ as spincoating SD1 on a metallic surface results in lower thicknesses than on fused silica. A slightly thicker layer also acts as a protection layer to prevent the aluminium from reacting with the polymer upon annealing at high temperatures (using $1 \mathrm{mg} \cdot \mathrm{mL}^{-1} \mathrm{SD} 1$ solution resulted in samples unfit for measurement). The sample was then annealed 6 minutes at a temperature of $150^{\circ} \mathrm{C}$ to drive any traces of solvent away and the rest of the alignment procedure was similar to the one used for the bare films. For the spincoating of the polymer layers, the solution concentrations were in some cases adjusted so as to adjust the thicknesses and therefore the cavity mode energy. The concentrations used in the non-aligned microcavities were: PFO at 19 mg.mL ${ }^{-1}$, F8BT at $20 \mathrm{mg} \cdot \mathrm{mL}^{-1}$ and F6T2 at $15 \mathrm{mg} \cdot \mathrm{mL}^{-1}$. The concentrations for the aligned microcavities were: PFO at $18 \mathrm{mg} \cdot \mathrm{mL}^{-1}$, F8BT at $18 \mathrm{mg} \cdot \mathrm{mL}^{-1}$, F6T2 at $13 \mathrm{mg} \cdot \mathrm{mL}^{-1}$.

\section{Optical Characterization}

The optical constants for the non-aligned and aligned films of PFO, F6T2 and F8BT were extracted using a J.A. Woollam ESM-300 ellipsometer. For each sample, 8 reflection-geometry measurements were performed with light incident from $45^{\circ}$ to $61^{\circ}$ (angles of incidence are quoted relative to the plane normal) together with a normal incidence $\left(0^{\circ}\right)$ transmission measurement, for the aligned films the measurement was performed at $\Phi=0^{\circ}$. The reflectivity maps obtained in Figure 1 were obtained using a home-built white light reflectivity setup. The microcavities were placed at the center of a stage with two independent rotating arms. A deuterium-halogen light source (DH-2000-DUV from Ocean Optics) was coupled into a fiber whose output was collimated onto a broadband polarizer (WP25M-UB from Thorlabs) and onto the sample (final spot size $1 \mathrm{~mm}$ ). The reflected light was then coupled into a second fiber placed onto the second arm and analyzed using a spectrometer (HRS 500, 150 
$\mathrm{g} / \mathrm{mm}$ grating with blazing wavelength at $300 \mathrm{~nm}$ ) and CCD (Pylon-2KB CCD from Princeton Instruments). In all cases, a neat aluminium mirror with known reflectivity was used as reference. The acquisition angle was varied by $0.5^{\circ}$ steps from 10 to $70^{\circ}$.

\section{Photoluminescence}

All the time-integrated PL measurements were performed on the same home-built setup. The fiber coupled to the white light source was disconnected and the first arm was positioned at $75^{\circ}$ relative to the sample plane normal. The pulsed laser beam from a supercontinuum white light laser (SuperK Extreme with its UV spectral extension unit Extend-UV, NKT Photonics) was used as excitation source and focused onto the sample (spot size $<1 \mathrm{~mm}$ ). The excitation energy was tuned according to the optical transitions, the incident power was kept low $(\leq 10 \mu W)$ with pulse widths of 20 to 30 ps and a repetition rate of $77.87 \mathrm{MHz}$. The broadband polarizer was placed on the collection arm, at a distance of $10 \mathrm{~cm}$ from the sample. Two nearly closed irises $(\sim 1 \mathrm{~cm})$ at a distance of $5 \mathrm{~cm}$ from each other were then placed before the coupling lens of the collection fiber in order to ensure that the collected light was emitted at the desired angle in the horizontal plane. The light was then analysed using the spectrometer and CCD described before using this time a $300 \mathrm{~g} / \mathrm{mm}$ grating blazed at a wavelength of $500 \mathrm{~nm}$. For the polymer films, the light was collected at normal incidence. For the microcavities the acquisition angle was varied by $1^{\circ}$ steps from -40 to $60^{\circ}$.

\section{Data Analysis}

The minima of the reflectivity maps shown in Figures 3 and 4 were analyzed using a leastsquare fitting algorithm for the eigenvalue problem:

$$
H_{q} \mathbf{v}_{\mathbf{i}, \mathbf{q}}=\omega_{i, q} \mathbf{v}_{\mathbf{i}, \mathbf{q}}
$$

where $H_{q}$ is an extension of the Agranovich/Hopfield Hamiltonian containing either 
one $^{22,62,63}$ (PFO, F6T2) or two excitonic resonances ${ }^{27}$ (F8BT):

$$
H_{q}=\left[\begin{array}{cccccc}
\omega_{\mathrm{cav}, q}+2 D_{q} & -i \frac{\Omega_{1, q}}{2} & -i \frac{\Omega_{2, q}}{2} & -2 D_{q} & -i \frac{\Omega_{1, q}}{2} & -i \frac{\Omega_{2, q}}{2} \\
i \frac{\Omega_{1, q}}{2} & \omega_{1} & 0 & -i \frac{\Omega_{1, q}}{2} & 0 & 0 \\
i \frac{\Omega_{2, q}}{2} & 0 & \omega_{2} & -i \frac{\Omega_{2, q}}{2} & 0 & 0 \\
2 D_{q} & -i \frac{\Omega_{1, q}}{2} & -i \frac{\Omega_{2, q}}{2} & -\omega_{\mathrm{cav}, q}-2 D_{q} & -i \frac{\Omega_{1, q}}{2} & -i \frac{\Omega_{2, q}}{2} \\
-i \frac{\Omega_{1, q}}{2} & 0 & 0 & i \frac{\Omega_{1, q}}{2} & -\omega_{1} & 0 \\
-i \frac{\Omega_{2, q}}{2} & 0 & 0 & i \frac{\Omega_{2, q}}{2} & 0 & -\omega_{2}
\end{array}\right],
$$

In the case of a single exciton oscillator, $H_{q}$ reduces to the usual $4 \times 4$ Hopfield-like USC matrix. ${ }^{10}$ In Eq. (2), (3), q is the in-plane wave vector, $\omega_{\text {cav }_{q}}$ the cavity mode energy, $\omega_{j}$ the frequency for the $j$-excitons, $\Omega_{j, q}$ is the associated Rabi frequency, and for a given angle $\theta: \Omega_{j, q}=\Omega_{j}(\theta)=\Omega_{0 j} \sqrt{\frac{\omega_{j}}{\omega_{\text {cav }}(\theta)}}$ where $\Omega_{0 j}$ is the Rabi frequency on resonance for the $j$-excitons. It was shown that in metal-organic semiconductor-metal cavities $\omega_{\text {cav }}(\theta)$ can be approximated by: ${ }^{10}$

$$
\omega_{\mathrm{cav}_{(\mathrm{TE}, \mathrm{TM}), q}}=\omega_{\mathrm{cav}_{(\mathrm{TE}, \mathrm{TM})}}(\theta)=\omega_{\mathrm{cav}}(0)\left(1-\frac{\sin ^{2}(\theta)}{n_{\mathrm{eff}}^{2}{ }_{(\mathrm{TE}, \mathrm{TM})}}\right)^{-\frac{1}{2}}
$$

where $n_{\mathrm{eff}}$ is,TM is polarization dependent. Finally, $D_{q}=\sum_{j} \frac{\Omega_{j, q}^{2}}{4 \omega_{j}}$ is the contribution of the squared magnetic vector potential.

In order to diagonalize $H$, the polariton annihilation operators $p_{i, q}=w_{i, q} a_{q}+\sum_{j} x_{i, j, q} b_{j, q}+$ $y_{i, q} a_{-q}^{\dagger}+\sum_{j} z_{i, j, q} b_{j,-q}^{\dagger}$ for $i \epsilon\{\mathrm{LP}, \mathrm{MP}, \mathrm{UP}\}$ are introduced, where $a_{q}$ and $a_{q}^{\dagger}$ respectively annihilate and create a photon at frequency $\omega_{\operatorname{cav}_{q}}, b_{j}$ and $b_{j}^{\dagger}$ respectively annihilate and create a $j$-exciton at frequency $\omega_{j}$. The terms $w, x, y$ and $z$ label, respectively, the photon, exciton, anomalous photon and anomalous exciton Hopfield coefficients. The eigenvalues of $H_{q}$ were fitted to the experimental results for each cavity, for both TE- and TM-polarization, using the $R$-minima in the $10-70^{\circ}$ range.

In order to minimize the number of fitting parameters and obtain meaningful results, 
only $\omega_{\mathrm{Cav}_{\mathrm{TE}, \mathrm{TM}}}(0), n_{\mathrm{eff}_{\mathrm{TE}, \mathrm{TM}}} \& \Omega_{01_{\mathrm{TE}, \mathrm{TM}}}$ were allowed to vary in fittings of the PFO and F6T2 cavities. Similarly, only $\omega_{\mathrm{Cav}_{\mathrm{TE}, \mathrm{TM}}}(0), n_{\mathrm{eff}}^{\mathrm{TE}, \mathrm{TM}}, \Omega_{01_{\mathrm{TE}, \mathrm{TM}}} \& \Omega_{02_{\mathrm{TE}, \mathrm{TM}}}$ were allowed to vary in the fitting of the F8BT cavities. For each exciton, the value of $\hbar \omega_{j}$ was set to be at the energy that corresponds to the mid-point of the integral oscillator strength for the corresponding

optical transition using $\int_{E_{\min }}^{\hbar \omega_{j}} \epsilon(\omega) d \omega=\frac{1}{2} \int_{E_{\min }}^{E_{\max }} \epsilon(\omega) d \omega$, where $\epsilon(\omega)$ is the extinction coefficient for $X_{j}$ in the $E_{\min }$ to $E_{\max }$ energy range.

\section{Supporting Information}

Optical constants of aligned and non-aligned LCCP films; Angular variation of s- and ppolarized transmittances at normal incidence for a thin film of aligned PFO; Experimental and analytical results for the ultrastrong coupling of non-aligned F8BT and PFO; TMR calculations

\section{Acknowledgement}

The authors thank Professor Moritz Riede for access to research facilities and Dr Richard Hamilton and Professor Ruidong Xia for fruitful discussions. They also acknowledge funding from the University of Oxford, from the UK Engineering and Physical Sciences Research Council and the Jiangsu Industrial Technology Research Institute. F.L.R. further thanks Wolfson College and Dr Simon Harrison for the award of a Wolfson Harrison UK Research Council Physics Scholarship. 


\section{For Table Of Contents Use Only}

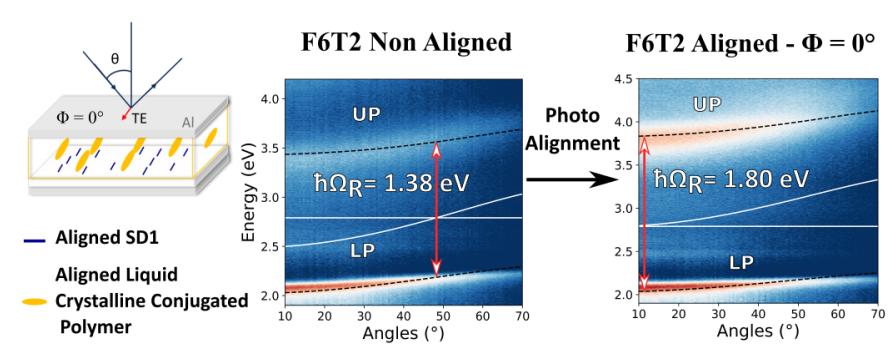

Figure 7: Table Of Contents Graphic illustrating the enhancement of the coupling following the alignement of liquid crystalline conjugated polymer chains inside a metallic microcavity. 


\section{References}

(1) Weisbuch, C.; Nishioka, M.; Ishikawa, A.; Arakawa, Y. Observation of the coupled exciton-photon mode splitting in a semiconductor quantum microcavity. Physical Review Letters 1992, 69, 3314-3317.

(2) Marks, R. N.; Halls, J. J. M.; Bradley, D. D. C.; Friend, R. H.; Holmes, A. B. The photovoltaic response in poly(p-phenylene vinylene) thin-film devices. Journal of Physics: Condensed Matter 1994, 6, 1379-1394.

(3) Alvarado, S.; Seidler, P.; Lidzey, D. G.; Bradley, D. D. C. Direct Determination of the Exciton Binding Energy of Conjugated Polymers Using a Scanning Tunneling Microscope. Physical Review Letters 1998, 81, 1082-1085.

(4) Plumhof, J. D.; Stöferle, T.; Mai, L.; Scherf, U.; Mahrt, R. F. Room-temperature Bose-Einstein condensation of cavity exciton-polaritons in a polymer. Nature Materials 2014, 13, 247-252.

(5) Daskalakis, K. S.; Maier, S. A.; Murray, R.; Kéna-Cohen, S. Nonlinear interactions in an organic polariton condensate. Nature Materials 2014, 13, 271-278.

(6) Lerario, G.; Ballarini, D.; Fieramosca, A.; Cannavale, A.; Genco, A.; Mangione, F.; Gambino, S.; Dominici, L.; De Giorgi, M.; Gigli, G.; Sanvitto, D. High-speed flow of interacting organic polaritons. Light: Science \& Applications 2017, 6, e16212-e16212.

(7) Zasedatelev, A. V.; Baranikov, A. V.; Urbonas, D.; Scafirimuto, F.; Scherf, U.; Stöferle, T.; Mahrt, R. F.; Lagoudakis, P. G. A room-temperature organic polariton transistor. Nature Photonics 2019, 13, 378-383.

(8) Lidzey, D. G.; Bradley, D. D. C.; Skolnick, M. S.; Virgili, T.; Walker, S.; Whittaker, D. M. Strong exciton-photon coupling in an organic semiconductor microcavity. Nature 1998, 395, 53-55. 
(9) Schwartz, T.; Hutchison, J. A.; Genet, C.; Ebbesen, T. W. Reversible Switching of Ultrastrong Light-Molecule Coupling. Physical Review Letters 2011, 106, 196405.

(10) Kéna-Cohen, S.; Maier, S. A.; Bradley, D. D. C. Ultrastrongly Coupled ExcitonPolaritons in Metal-Clad Organic Semiconductor Microcavities. Advanced Optical Materials 2013, 1, 827-833.

(11) Gambino, S.; Mazzeo, M.; Genco, A.; Di Stefano, O.; Savasta, S.; Patanè, S.; Ballarini, D.; Mangione, F.; Lerario, G.; Sanvitto, D.; Gigli, G. Exploring Light-Matter Interaction Phenomena under Ultrastrong Coupling Regime. ACS Photonics 2014, 1, $1042-1048$.

(12) Mazzeo, M.; Genco, A.; Gambino, S.; Ballarini, D.; Mangione, F.; Di Stefano, O.; Patanè, S.; Savasta, S.; Sanvitto, D.; Gigli, G. Ultrastrong light-matter coupling in electrically doped microcavity organic light emitting diodes. Applied Physics Letters 2014, 104, 233303.

(13) Suzuki, M.; Nishiyama, K.; Kani, N.; Yu, X.; Uzumi, K.; Funahashi, M.; Shimokawa, F.; Nakanishi, S.; Tsurumachi, N. Observation of ultrastrong-coupling regime in the Fabry-Pérot microcavities made of metal mirrors containing Lemke dye. Applied Physics Letters 2019, 114, 191108.

(14) Liu, B.; Rai, P.; Grezmak, J.; Twieg, R. J.; Singer, K. D. Coupling of exciton-polaritons in low - Q coupled microcavities beyond the rotating wave approximation. Physical Review B 2015, 92, 155301.

(15) Liu, B.; Crescimanno, M.; Twieg, R. J.; Singer, K. D. Dispersion of Third-Harmonic Generation in Organic Cavity Polaritons. Advanced Optical Materials 2019, 1801682.

(16) Frisk Kockum, A.; Miranowicz, A.; De Liberato, S.; Savasta, S.; Nori, F. Ultrastrong coupling between light and matter. Nature Reviews Physics 2019, 1, 19-40. 
(17) Forn-Díaz, P.; Lamata, L.; Rico, E.; Kono, J.; Solano, E. Ultrastrong coupling regimes of light-matter interaction. Reviews of Modern Physics 2019, 91, 025005.

(18) Askenazi, B.; Vasanelli, A.; Todorov, Y.; Sakat, E.; Greffet, J.-J.; Beaudoin, G.; Sagnes, I.; Sirtori, C. Midinfrared Ultrastrong Light-Matter Coupling for THz Thermal Emission. ACS Photonics 2017, 4, 2550-2555.

(19) Yoshihara, F.; Fuse, T.; Ao, Z.; Ashhab, S.; Kakuyanagi, K.; Saito, S.; Aoki, T.; Koshino, K.; Semba, K. Inversion of Qubit Energy Levels in Qubit-Oscillator Circuits in the Deep-Strong-Coupling Regime. Physical Review Letters 2018, 120, 183601.

(20) Bayer, A.; Pozimski, M.; Schambeck, S.; Schuh, D.; Huber, R.; Bougeard, D.; Lange, C. Terahertz Light-Matter Interaction beyond Unity Coupling Strength. Nano Letters 2017, 17, 6340-6344.

(21) Benz, F.; Schmidt, M. K.; Dreismann, A.; Chikkaraddy, R.; Zhang, Y.; Demetriadou, A.; Carnegie, C.; Ohadi, H.; de Nijs, B.; Esteban, R.; Aizpurua, J.; Baumberg, J. J. Single-molecule optomechanics in "picocavities". Science (New York, N.Y.) 2016, 354, 726-729.

(22) Ciuti, C.; Bastard, G.; Carusotto, I. Quantum vacuum properties of the intersubband cavity polariton field. Physical Review B 2005, 72, 115303.

(23) George, J.; Wang, S.; Chervy, T.; Canaguier-Durand, A.; Schaeffer, G.; Lehn, J.-M.; Hutchison, J. A.; Genet, C.; Ebbesen, T. W. Ultra-strong coupling of molecular materials: spectroscopy and dynamics. Faraday Discussions 2015, 178, 281-294.

(24) Tropf, L.; Gather, M. C. Investigating the Onset of the Strong Coupling Regime by Fine-Tuning the Rabi Splitting in Multilayer Organic Microcavities. Advanced Optical Materials 2018, 6, 1800203. 
(25) Barachati, F.; Simon, J.; Getmanenko, Y. A.; Barlow, S.; Marder, S. R.; KénaCohen, S. Tunable Third-Harmonic Generation from Polaritons in the Ultrastrong Coupling Regime. ACS Photonics 2018, 5, 119-125.

(26) Campoy-Quiles, M.; Ferenczi, T.; Agostinelli, T.; Etchegoin, P. G.; Kim, Y.; Anthopoulos, T. D.; Stavrinou, P. N.; Bradley, D. D. C.; Nelson, J. Morphology evolution via self-organization and lateral and vertical diffusion in polymer:fullerene solar cell blends. Nature Materials 2008, 7, 158-164.

(27) Le Roux, F.; Bradley, D. D. C. Conformational control of exciton-polariton physics in metal-poly(9,9-dioctylfluorene)-metal cavities. Physical Review B 2018, 98, 195306.

(28) Perevedentsev, A.; Chander, N.; Kim, J.-S.; Bradley, D. D. C. Spectroscopic properties of poly(9,9-dioctylfluorene) thin films possessing varied fractions of $\beta$-phase chain segments: enhanced photoluminescence efficiency via conformation structuring. Journal of Polymer Science Part B: Polymer Physics 2016, 54, 1995-2006.

(29) Kéna-Cohen, S.; Davanço, M.; Forrest, S. R. Strong exciton-photon coupling in an organic single crystal microcavity. Physical Review Letters 2008, 101.

(30) Virgili, T.; Lidzey, D.; Grell, M.; Walker, S.; Asimakis, A.; Bradley, D. Completely polarized photoluminescence emission from a microcavity containing an aligned conjugated polymer. Chemical Physics Letters 2001, 341, 219-224.

(31) Campoy-Quiles, M.; Etchegoin, P. G.; Bradley, D. D. C. On the optical anisotropy of conjugated polymer thin films. Physical Review B 2005, 72, 045209.

(32) Hertzog, M.; Rudquist, P.; Hutchison, J. A.; George, J.; Ebbesen, T. W.; Börjesson, K. Voltage-Controlled Switching of Strong Light-Matter Interactions using Liquid Crystals. Chemistry - A European Journal 2017, 23, 18166-18170. 
(33) Gao, W.; Li, X.; Bamba, M.; Kono, J. Continuous transition between weak and ultrastrong coupling through exceptional points in carbon nanotube microcavity exciton-polaritons. Nature Photonics 2018, 12, 362-367.

(34) Litinskaya, M.; Reineker, P.; Agranovich, V. M. Exciton-polaritons in a crystalline anisotropic organic microcavity. physica status solidi (a) 2004, 201, 646-654.

(35) Balagurov, D. B.; La Rocca, G. C. Organic microcavities with anisotropic optically active materials. physica status solidi (c) 2004, 1, 518-521.

(36) Grell, M.; Bradley, D. D. C.; Inbasekaran, M.; Woo, E. P. A glass-forming conjugated main-chain liquid crystal polymer for polarized electroluminescence applications. $A d$ vanced Materials 1997, 9, 798-802.

(37) Grell, M.; Redecker, M.; Whitehead, K. S.; Bradley, D. D. C.; Inbasekaran, M.; Woo, E. P. Monodomain alignment of thermotropic fluorene copolymers. Liquid Crystals 1999, 26, 1403-1407.

(38) Whitehead, K. S.; Grell, M.; Bradley, D. D. C.; Jandke, M.; Strohriegl, P. Highly polarized blue electroluminescence from homogeneously aligned films of poly $(9,9-$ dioctylfluorene). Applied Physics Letters 2000, 76, 2946-2948.

(39) Dyreklev, P.; Berggren, M.; Inganäs, O.; Andersson, M. R.; Wennerström, O.; Hjertberg, T. Polarized electroluminescence from an oriented substituted polythiophene in a light emitting diode. Advanced Materials 1995, 7, 43-45.

(40) Cimrová, V.; Remmers, M.; Neher, D.; Wegner, G. Polarized light emission from LEDs prepared by the Langmuir-Blodgett technique. Advanced Materials 1996, 8, 146-149.

(41) Hagler, T. W.; Pakbaz, K.; Voss, K. F.; Heeger, A. J. Enhanced order and electronic delocalization in conjugated polymers oriented by gel processing in polyethylene. Physical Review B 1991, 44, 8652-8666. 
(42) Zheng, Z.; Yim, K.; Saifullah, M.; Welland, M. E.; Friend, R. H.; Kim, J.-S.; S., H. W. T. Uniaxial Alignment of Liquid-Crystalline Conjugated Polymers by Nanoconfinement. 2007 ,

(43) Whitehead, K.; Grell, M.; Bradley, D.; Inbasekaran, M.; Woo, E. Polarized emission from liquid crystal polymers. Synthetic Metals 2000, 111-112, 181-185.

(44) Schmid, S. A.; Yim, K. H.; Chang, M. H.; Zheng, Z.; Huck, W. T. S.; Friend, R. H.; Kim, J. S.; Herz, L. M. Polarization anisotropy dynamics for thin films of a conjugated polymer aligned by nanoimprinting. Physical Review B 2008, 77, 115338.

(45) Lee, D.-M.; Lee, Y.-J.; Kim, J.-H.; Yu, C.-J. Birefringence-dependent linearly-polarized emission in a liquid crystalline organic light emitting polymer. Optics Express 2017, 25,3737 .

(46) Misaki, M.; Chikamatsu, M.; Yoshida, Y.; Azumi, R.; Tanigaki, N.; Yase, K.; Nagamatsu, S.; Ueda, Y. Highly efficient polarized polymer light-emitting diodes utilizing oriented films of $\beta$-phase poly(9,9-dioctylfluorene). Applied Physics Letters 2008, 93, 023304 .

(47) Sirringhaus, H.; Wilson, R. J.; Friend, R. H.; Inbasekaran, M.; Wu, W.; Woo, E. P.; Grell, M.; Bradley, D. D. C. Mobility enhancement in conjugated polymer field-effect transistors through chain alignment in a liquid-crystalline phase. Applied Physics Letters 2000, 77, 406-408.

(48) Seki, T. New strategies and implications for the photoalignment of liquid crystalline polymers. Polymer Journal 2014, 46, 751-768.

(49) Li, X.; Kozenkov, V. M.; Yeung, F. S.-Y.; Xu, P.; Chigrinov, V. G.; Kwok, H.-S. LiquidCrystal Photoalignment by Super Thin Azo Dye Layer. Japanese Journal of Applied Physics 2006, 45, 203-205. 
(50) Ma, L.-L.; Li, S.-S.; Li, W.-S.; Ji, W.; Luo, B.; Zheng, Z.-G.; Cai, Z.-P.; Chigrinov, V.; Lu, Y.-Q.; Hu, W.; Chen, L.-J. Rationally Designed Dynamic Superstructures Enabled by Photoaligning Cholesteric Liquid Crystals. Advanced Optical Materials 2015, 3, 1691-1696.

(51) Zhang, H.; Ma, L.; Zhang, Q.; Shi, Y.; Fang, Y.; Xia, R.; Hu, W.; Bradley, D. D. C. Advanced Optical Materials - In Press 2020,

(52) Ma, L.-L.; Tang, M.-J.; Hu, W.; Cui, Z.-Q.; Ge, S.-J.; Chen, P.; Chen, L.-J.; Qian, H.; Chi, L.-F.; Lu, Y.-Q. Smectic Layer Origami via Preprogrammed Photoalignment. Advanced Materials 2017, 29, 1606671.

(53) Sanvitto, D.; Kéna-Cohen, S. The road towards polaritonic devices. Nature Materials 2016, 15, 1061-1073.

(54) Solnyshkov, D.; Bleu, O.; Malpuech, G. All optical controlled-NOT gate based on an exciton-polariton circuit. Superlattices and Microstructures 2015, 83, 466-475.

(55) Espinosa-Ortega, T.; Liew, T. C. H. Complete architecture of integrated photonic circuits based on and and not logic gates of exciton polaritons in semiconductor microcavities. Physical Review B 2013, 87, 195305.

(56) Campoy-Quiles, M.; Heliotis, G.; Xia, R.; Ariu, M.; Pintani, M.; Etchegoin, P.; Bradley, D. D. C. Ellipsometric Characterization of the Optical Constants of Polyfluorene Gain Media. Advanced Functional Materials 2005, 15, 925-933.

(57) Campoy-Quiles, M.; Alonso, M. I.; Bradley, D. D. C.; Richter, L. J. Advanced Ellipsometric Characterization of Conjugated Polymer Films. Advanced Functional Materials 2014, 24, 2116-2134.

(58) Campoy-Quiles, M.; Etchegoin, P. G.; Bradley, D. D. C. On the optical anisotropy of conjugated polymer thin films. Physical Review B 2005, 72, 045209. 
(59) Tropf, L.; Dietrich, C. P.; Herbst, S.; Kanibolotsky, A. L.; Skabara, P. J.; Würthner, F.; Samuel, I. D. W.; Gather, M. C.; Höfling, S. Influence of optical material properties on strong coupling in organic semiconductor based microcavities. Applied Physics Letters 2017, 110, 153302 .

(60) Rothe, C.; Galbrecht, F.; Scherf, U.; Monkman, A. The $\beta$-Phase of Poly $(9,9$ dioctylfluorene) as a Potential System for Electrically Pumped Organic Lasing. Advanced Materials 2006, 18, 2137-2140.

(61) Valyukh, I.; Arwin, H.; Chigrinov, V.; Valyukh, S. UV-induced in-plane anisotropy in layers of mixture of the azo-dyes SD-1/SDA-2 characterized by spectroscopic ellipsometry. physica status solidi (c) 2008, 5, 1274-1277.

(62) Agranovich, V. M. Opt Spektrosk. 1957, 2.

(63) Hopfield, J. J. Theory of the Contribution of Excitons to the Complex Dielectric Constant of Crystals. Physical Review 1958, 112, 1555-1567.

(64) Economou, E. N. Surface Plasmons in Thin Films. Physical Review 1969, 182, 539-554.

(65) Litinskaya, M.; Agranovich, V. M. Polariton trap in microcavities with metallic mirrors. Journal of Physics: Condensed Matter 2012, 24, 015302.

(66) Kavokin, A. V. Motional narrowing of inhomogeneously broadened excitons in a semiconductor microcavity: Semiclassical treatment. Physical Review B - Condensed Matter and Materials Physics 1998, 5\%, 3757-3760.

(67) Houdré, R.; Stanley, R. P.; Ilegems, M. Vacuum-field Rabi splitting in the presence of inhomogeneous broadening: Resolution of a homogeneous linewidth in an inhomogeneously broadened system. Physical Review A - Atomic, Molecular, and Optical Physics 1996, 53, 2711-2715. 
(68) Yuen-Zhou, J.; Saikin, S. K.; Zhu, T.; Onbasli, M. C.; Ross, C. A.; Bulovic, V.; Baldo, M. A. Plexciton Dirac points and topological modes. Nature Communications 2016, 7 .

(69) Perevedentsev, A.; Sonnefraud, Y.; Belton, C. R.; Sharma, S.; Cass, A. E. G.; Maier, S. A.; Kim, J.-S.; Stavrinou, P. N.; Bradley, D. D. C. Dip-pen patterning of poly(9,9-dioctylfluorene) chain-conformation-based nano-photonic elements. Nature Communications 2015, 6, 5977.

(70) Hamilton, I.; Chander, N.; Cheetham, N. J.; Suh, M.; Dyson, M.; Wang, X.; Stavrinou, P. N.; Cass, M.; Bradley, D. D. C.; Kim, J.-S. Controlling Molecular Conformation for Highly Efficient and Stable Deep-Blue Copolymer Light-Emitting Diodes. ACS Applied Materials $\mathscr{E}$ Interfaces 2018, 10, 11070-11082.

(71) Scafirimuto, F.; Urbonas, D.; Scherf, U.; Mahrt, R. F.; Stöferle, T. Room-Temperature Exciton-Polariton Condensation in a Tunable Zero-Dimensional Microcavity. ACS Photonics 2018, 5, 85-89.

(72) Eizner, E.; Brodeur, J.; Barachati, F.; Sridharan, A.; Kéna-Cohen, S. Organic Photodiodes with an Extended Responsivity Using Ultrastrong Light-Matter Coupling. ACS Photonics 2018, 5, 2921-2927.

(73) Rajendran, S. K.; Wei, M.; Ohadi, H.; Ruseckas, A.; Turnbull, G. A.; Samuel, I. D. W. Low Threshold Polariton Lasing from a Solution-Processed Organic Semiconductor in a Planar Microcavity. Advanced Optical Materials 2019, 7, 1801791.

(74) Wei, M.; Rajendran, S. K.; Ohadi, H.; Tropf, L.; Gather, M. C.; Turnbull, G. A.; Samuel, I. D. W. Low-threshold polariton lasing in a highly disordered conjugated polymer. Optica 2019, 6, 1124. 